\title{
Anthony GLINOER, La Bohème. Une figure de l'imaginaire social
}

Montréal, Les Presses de l'université de Montréal, 2018, 242 pages

\section{Olivier Desouches}

\section{(2) OpenEdition}

\section{Journals}

Édition électronique

URL : https://journals.openedition.org/questionsdecommunication/21526

DOI : 10.4000/questionsdecommunication.21526

ISSN : 2259-8901

Éditeur

Presses universitaires de Lorraine

Édition imprimée

Date de publication : 31 décembre 2019

Pagination : 323-325

ISBN : 9782814305632

ISSN : 1633-5961

Référence électronique

Olivier Desouches, «Anthony gLINOER, La Bohème. Une figure de l'imaginaire social », Questions de communication [En ligne], 36 | 2019, mis en ligne le 31 décembre 2019, consulté le 25 juin 2022. URL http://journals.openedition.org/questionsdecommunication/21526 ; DOI : https://doi.org/10.4000/ questionsdecommunication.21526 
Dans le premier article, Proust relate le meurtre d'une amie de ses parents, Mme van Blarenberghe, épouse du président de la Compagnie des chemins de fer de l'Est tuée par son propre fils (pp. 229-234). Cet article est de fait une réponse à un autre publié quelques jours plus tôt sur le même événement, écrit par un journaliste professionnel et intitulé « Un drame de la folie ». L'article du journaliste respecte les conventions habituelles $d u$ fait divers, combinant relation objective et théâtralisation. Le ton en est parfaitement donné par son incipit : « Le magnifique hôtel de la famille van Blarenberghe, 48 , rue de la Bienfaisance, a été hier le théatre d'un drame terrible. Un fils a tué sa mère et s'est ensuite suicidé »s. Proust relate lui aussi l'événement de manière dramatique, mais utilise un registre subjectif et littéraire. II commence par se mettre en scène, se rappelant une lettre du jeune homme à laquelle il doit répondre avant de découvrir la nouvelle dans Le Figaro. II restitue ensuite l'événement comme une tragédie littéraire, citant $\mathbb{E}$ dipe et Jocaste ou les héros de William Shakespeare, Fiodor Dostoïevski et Léon Tolstoï. Proust confère enfin à l'événement une portée universelle, concluant avec un paragraphe annonçant le futur thème récurrent dans son œuvre de la culpabilité envers ceux que l'on aime. De même, l'article «Impressions de route en automobile » est justement célèbre pour ses références autobiographiques comme pour l'annonce de thèmes qui en seront repris dans La Recherche : promenade en automobile dans la campagne normande, cathédrales médiévales, changements de perspective liés à la vitesse, allusions à John Ruskin, au chauffeur et amant Alfred Agostinelli... Yuri Cerqueira dos Anjos montre que cet article parait au moment où Paris reçoit l'Exposition décennale de l'automobile, abondamment relayée par la presse, et notamment par Le Figaro (pp. 134- 144). Ce souci d'ancrer ses articles dans l'actualité se retrouve dans les prépublications de 1912. Par rapport aux textes figurant dans Du côté de chez Swann, les articles sont introduits par des déictiques et références temporelles. Ainsi le texte « Épines blanches, épines roses » s'ouvre-t-il sur les mots suivants: «Je lisais, l'autre jour,à propos de cet hivers relativement doux - qui s'achève aujourd'hui » (p. 293).

L'ouvrage a été présenté lors d'un après-midi d'étude le 10 février 2018 à la Bibliothèque nationale de France (Proust et la presse dans le cadre du cycle, Les Écrivains et la presse, Blog BnF, en ligne). Outre plusieurs contributions d'universitaires faisant référence dans les études proustiennes, ce colloque a permis de présenter les ressources disponibles en ligne sur Gallica, le portail de la Bibliothèque nationale de France et de ses bibliothèques partenaires. Les manuscrits du fonds Proust sont désormais inventoriés et numérisés. L'équipe de l'Institut des textes et manuscrits modernes (CNRS,
École normale supérieure) a développé un portail raisonné permettant d'y accéder directement. Sur son site personnel, Yuri Cerqueira dos Anjos propose également des articles de presse directement accessibles. L'ensemble de ces ressources numériques est présenté sur le blog Gallica (" Proust et la presse, présentation des collections dans Gallica », Le Blog Gallica, en ligne.)

Vincent Hecquet

Institut national de la statistique et des études économiques F-92120

vincenthecquet[at]ec.europa.eu

\section{Anthony GLINOER, La Bohème. Une figure de Pimaginaire social}

Montréal, Les Presses de l'université de Montréal, 2018, 242 pages

« Nous avons chacune et chacun une idée de ce qu'est la bohème et de qui est bohème » ( $p . \mid \mathrm{I})$ : d'un côté, hommes de lettres (Gérard de Nerval), artistes et «l'intelligentsia prolétaroïde » (terme que Pierre Bourdieu emprunte à Max Weber, Les Règles de l'art. Genèse et structure du champ littéraire, Paris, Éd. Le Seuil, 1992) d'étudiants qui passent de 2500 à 12000 au cours du XIX' siècle en France ; de l'autre, surconsommation de tabac, d'alcool et de drogues, jeunesse, vagabondage, pauvreté, paresse, idées politiques souvent radicales. Elle date de la France de 1830 et de la bataille d'Hernani, polémique née des représentations de la pièce deVictor Hugo opposant la nouvelle génération de ses partisans (comme Théophile Gautier) et, plus largement, les adeptes du théâtre romantique aux classiques.

« Nous nous représentons les lieux où se déploient l'existence du bohème » dans ses ateliers, la Brasserie des Martyrs - fréquentée par Manet, Daudet ou Courbet sous le Second Empire -, le Quartier latin, le cabaret Au Lapin Agile ou le Moulin de la Galette. Ainsi, l'affiche du Chat noir, du nom du célèbre cabaret de la butte Montmartre, est-elle certainement une des reproductions les plus souvent associées à la bohème de la fin du XIXe siècle. La bohème est ici retracée comme une figure emblématique des imaginaires sociaux et de nos représentations de la vie d'artistes comme d'écrivains, des Scènes de la vie de Bohème (Henri Murger, Paris, Fayard, 1850) en passant par son adaptation à l'opéra par Glacomo Puccini en 1896, I'un « des plus connus et des plus joués du répertoire » (p. 15) qui se déroule dans la rue, une mansarde ou un café - car « dans la mansarde, le bohème est seul face à son indigence ; au café il est vu, entendu et reconnu » (pp. 221-222) - , jusqu'à la chanson qu'Aznavour associe en 1965 aux peintres, depuis aux bobos et à la « classe créative » dont 
le professeur d'études urbaines Richard Florida souligna l'émergence en 2002.

Du « cercle des poètes zutiques » auquel participèrent Arthur Rimbaud et Paul Verlaine aux dadaïstes jusqu'à Greenwich Village, « l'imaginaire de la bohème a essaimé partout dans le monde occidental » (p. I3) pour Anthony Glinoer. L'auteur de cet essai, professeur à l'université de Sherbrooke, titulaire de la Chaire de recherche du Canada sur l'histoire de l'édition et la sociologie du littéraire, est spécialiste de l'étude des représentations de la vie littéraire. Malgré le désir de singularité propre à la vie d'artiste, le bohème vit en communauté, en réseau ou en groupe, qu'il s'agisse des buveurs d'eau ou des hydropathes. S'érigeant en contreculture d'un point de vue idéologique, la bohème « comme figure de l'imaginaire social, présente deux caractéristiques remarquables » (p. 23) : nombreuses sont les querelles d'interprétations dont elle est l'objet alors qu'elle en est pourtant la principale pourvoyeuse.

Le premier chapitre est consacré à l'« Émergence » de la notion entre 1830 et I 880 tandis que le deuxième, «Altérités 》, définit le bohème par sa rupture avec le bourgeois, qu'il soit propriétaire, cafetier, éditeur ou marchand de tableaux, même quand il ne peut être systématiquement associé aux bas-fonds. En fait, il n'est ni le dandy théorisé par Barbey d'Aurevilly, ni un bohémien, terme « auquel on préfère aujourd'hui les vocables de tsiganes, manouches ou Roms 》 (p. 82). Qu'elles soient risettes, lorettes, cocottes, lolottes, actrices et prostituées, les femmes ne sont que de passage, aux quelques exceptions près que sont Louise Colet, Flora Tristan et Nina deVillard.

L'objet du chapitre «Trajectoires » est « de comprendre comment ont été inventés un certain nombre de héros de la bohème en suivant leur biographie sociale » (p.9l). Qu'il s'agisse « des vagabonds, des soldats licenciés, des forçats sortis du bagne, des galériens en rupture de ban, des filous, des charlatans, des lazzaroni, des pickpockets, des escamoteurs, des joueurs, des souteneurs, des tenanciers de maisons publiques, des portefaix, des écrivassiers, des joueurs d'orgue, des chiffonniers, des rémouleurs, des rétameurs, des mendiants 》 (p. 50) énumérés par Karl Marx dans Le 18 Brumaire de Louis Bonaparte, l'auteur les répartit entre archi-bohème emblématique - comme Privat d'Anglemont, le noctambule Fernand Desnoyers ou le pilier de café à ses heures graveur Marcellin Desboutin -, réfractaires - le socialiste révolutionnaire Eugène Vermersch - et renégats - l'Académicien Jean Richepin.

L'avant-dernier chapitre examine les «Écritures 》 de la bohème, à savoir des poèmes, des romans tels que
La dernière Aldini de George Sand à qui revient « la première occurrence du terme "bohème" dans son acception moderne » (p. 208), Le Bachelier de Jules Vallès, Manette Salomon de Jules et Edmond de Goncourt et L'Éuvre d'Émile Zola, ou de la presse. Celle-ci (La Vache enragée, L'Hydropathe, Chat noir) est d'ailleurs étudiée plus spécifiquement dans l'ouvrage Vie de bohème et petite presse du XXXe siècle. Sociabilité littéraire ou solidarité journalistique? (Alain Vaillant, Yoan Vérilhac, Paris, Presses universitaires de Paris-Nanterre, 2018), où une contribution d'Anthony Glinoer et Vincent Laisney retrace l'histoire de la Brasserie des Martyrs comme lieu de sociabilité, de Louis Philippe à Napoléon III. La bohème se complait dans les souvenirs littéraires d'Alphonse Daudet (Trente ans de Paris: à travers ma vie et mes lives) ou d'Emile Goudeau (Dix ans de bohème).

Enfin, le chapitre « Transferts» documente le « déploiement transnational et transhistorique du phénomène de la bohème qui n'est jamais tout à fait parvenu à son terme » (p. 24I) entre I850 et I900 avec son appellation propre (scapigliatura) en Italie. À Varsovie comme à Melbourne, de Buenos Aires et Rio de Janeiro à Montréal, elle ne connait pas de frontières : en attestent les illustrations du peintre norvégien Edvard Munch ou du livre La santa bohemia de l'émigré letton à Madrid Ernesto Bark. Le magnétisme de Paris, terminus de retours d'Europe, ne sera que croissant après la Première Guerre mondiale car, « en matière de bohème, rien ne s'arrête en 1880 ni même en 1914 [...]. D'autres figures de l'imaginaire social en ont d'ailleurs hérité de nombreux traits, tels les beatniks, les hippies et les hipsters » (p. 259).

Sans doute ce final aurait pu se prolonger avec la distinction canonique que le sociologue Robert $\mathrm{E}$. Park, l'un des fondateurs de l'école de Chicago, établit entre deux quartiers de cette ville, Bohemia (La Bohème qui est une colonie d'artistes) et Hobohemia (la Cloche) à la suite de la publication en 1923 de Le Hobo. Sociologie du sans-abri de Nels Anderson. «C'est là que s'entasse cette population vagabonde d'hommes sans foyer, homeless men, venue du dehors et qui y retourne, les Hobos, d'où l'on a tiré le mot: Hobohemia » relate Maurice Halbwachs dans son article de 1932 («Chicago, expérience ethnique », Annales d'histoire économique et sociale, 13; p. 20). Ce n'est plus le « bourgeois possédant, lourd et enraciné » mais le « bohème, léger, libre et nomade » qui incarne « Le nouvel esprit du capitalisme » (Luc Botanski, Eve Chiapello, Le nouvel esprit du capitalisme, Paris, Gallimard, 1999). De même, dans sa thèse Les Intellectuels précaires, genèses et réalités d'une figure critique soutenue en 2015 à l'École des hautes études en sciences sociales, Cyprien Tasset articule l'imaginaire social de la bohème au 
XIX siècle autour de trois répertoires: la perspective de la révolte, à la suite de Jules Vallès ; le désenchantement qui consiste à dénoncer la faible valeur artistique de la bohème, et donc à la soupçonner d'être mystificatrice ; les séductions d'un mode de ville stylisé, dans l'héritage d'Henri Murger comme en atteste la mise en généralité par les actuels bobos de la gentrification.

Olivier Desouches

Arènes, université Rennes 2, F-35043 olivier.desouches[at]univ-rennes2.fr

\section{Jean-Marc Gouanvic, Hard-Boiled fiction et série noire. Les métamorphoses du roman policier anglo-américain en français (1945-1960)}

Paris, Classiques Garnier, coll.Translatio, 20 18,28I pages

L'ouvrage produit par Jean-Marc Gouanvic est une étude croisant analyses littéraires et sociologiques sur les processus de traduction des récits - ici, les romans policiers « hard boiled», étasuniens au premier chef - d'une langue et d'une culture vers une autre. La structure du volume est mosäque, peut-être parce que synthétisant des textes et réflexions produites en des occasions diverses. Pour donner un aperçu des contenus du volume, celui-ci comporte des développements qui balisent la place de la « Série noire » de Gallimard dans le champ littéraire français. D'autres analyses qu'on peut encore qualifier de synthétiques, questionnent ce qui advient quand un corpus littéraire très marqué par une culture et une histoire nationale, migre par traduction, vers une autre société et d'autres lectorats. Une autre série de développements, occupant près des trois quarts du livre peut être définie comme plus thématique, même si elle sert de façon cohérente la réflexion générale de l'ouvrage.ll s'agit alors d'éclairer le statut de composantes (l'érotique) ou de registres d'expression (l'argot), mais bien plus dans la seconde moitié du livre, de rentrer finement dans le détail des traductions de Dashiell Hammett, Raymond Chandler, Chester Himes.

En espérant que l'adjectif ne fasse pas fuir les lecteurs potentiels on peut qualifier le livre de Jean-Marc Gouanvic d'érudit ou de savant. L'auteur est plus que familier de l'histoire et des protagonistes de la «Série noire 》, plus largement du sous-champ des littératures policières et d'aventures (p. 23-36) avec la concurrence féroce entre Gallimard, les Fleuve Noir (devenus Éditions du Fleuve) et les Presses de la Cité dans les années 1950. La complexité des rapports entre Marcel Duhamel et la direction de Gallimard, la composition et les méthodes de travail du pool de traducteurs sont rappelées de façon très précise et utile. L'auteur est aussi, d'abord, une référence sur les pratiques et enjeux sociaux de la traduction, auxquels il a consacré plusieurs volumes. C'est d'ailleurs ce qui fait à la fois la grande force du volume et une certaine rugosité de lecture. Les développements sur les auteurs et leurs traductions comportent de nombreuses pages mettant en vis-à-vis texte original et traduction française, rendant visibles par des soulignés ou des italiques, les coupes ou les traductions pour le moins audacieuses ou peu encombrées de coller au texte original. Ces longues citations de romans policiers ne sauraient être présentées comme se lisant « comme des romans policiers 》, elles n'en sont pas moins éclairantes, et à bien des égards, passionnantes pour qui veut se plonger dans les coulisses des jeux de la traduction et comprendre le «métier » du traducteur à la fois comme intelligence de deux langues, mais aussi comme capacité à s'adapter à un horizon de réception, à ce que l'éditeur imagine, à tort ou à raison des attentes du public. On peut mentionner, comme une des illustrations abouties sur ce plan, le chapitre (pp. |8|-220) qui décortique les conditions de l'abrégement de «The long Goodbye » de Raymond Chandler. Un point central de l'analyse de Jean-Marc Gouanvic tient ici dans la caractérisation d'une sensibilité de beaucoup de récits « hard-boiled » comme participant d'une illusio « puritaine », d'une culpabilité sociale généralisée (pp. 51-64) dans une société livrée à l'injustice, à la corruption et à des forces immorales. Un des effets de la traduction en Série noire, où Marcel Duhamel insiste sur la quête d'un style aventureux et ludique, va être un maquillage partiel de cette dimension. L'usage dans les traductions française d'un argot (pp. 95-132) - souvent absent des textes originaux - sera l'un des outils de cette transformation, donnant aux textes un ton plus ludique, plus savoureux (p. 132). Aussi le processus de traduction aboutit-il à habiller les récits anglophones d'un certain nombre d'attributs des traditions du roman populaire et d'aventures propre à la France.

L'objectif de Jean-Marc Gouanvic est aussi de sinscrire dans une approche sociologique de la traduction qui sinspire de Pierre Bourdieu. Approche sociologique : « cela signifie qu'est considérée comme capitale la relation entre la structure institutionnelle du champ ou prennent place les romans publiées, les agents actifs dans le même champ, les contenus thématiques et discursifs des romans et l'effet intériorisé du public lecteur » (p.9). Ayant personnellement contribué à une approche sociologique des littératures d'espionnage (Érik Neveu, Lidéologie dans le roman d'espionnage, Paris, Presses de Sciences Po, 1984), inspirée de Bourdieu, jai souvent plaidé - avec un remarquable insuccès - pour que les chercheurs ne soient pas sommés de choisir un camp, de 